\title{
超导量子计算: 长退相干量子比特发展之路
}

\author{
金贻荣*，郑东宁 \\ 中国科学院物理研究所, 凝聚态国家实验室, 超导国家重点实验室, 北京 100190 \\ * 联系人, E-mail: jyr-king@iphy.ac.cn
}

2017-06-23 收稿, 2017-07-18 修回, 2017-07-18 接受, 2017-09-27 网络版发表

国家重点基础研究发展计划(2014CB921401)和国家自然科学基金(11374344, 11674376)资助

\begin{abstract}
摘要超导量子计算是基于约瑟夫森结电路的固态量子计算技术方案. 由于其易于扩展、耦合与操控等特点, 已成为最有可能实现实用量子计算机/模拟机的候选方案之一. 由于固态器件复杂的电磁环境, 超导量子比特一 直受到退相干时间不足的制约. 本文简要介绍了超导量子比特的基本结构并重点论述了近期发展出的几种新型长 退相干时间超导量子比特, 包括Transmon/Xmon, Fluxonium和C-shunt Flux qubit等, 整理了这几个不同设计方案 在抑制电荷、磁通和准粒子等几种主要噪声来源方面做出的探索和贡献. 最后, 对几种主要的设计思想做了简要 的归纳总结，以期对未来进一步发展提供参考和依据.
\end{abstract}

关键词量子计算, 超导量子比特, 退相干时间, 约瑟夫森结

从 20 世纪 80 年代Feynman ${ }^{[1]}$ 提出利用量子力学基 本原理来更好地模拟/解决物理问题的概念以来, 量 子计算技术一直是人们关注的一项极具革命性和挑 战性的前沿课题. 尽管早在 20 世纪90年代, 有效的量 子算法, 包括Peter Shor的质因数分解算法 ${ }^{[2]}$ 和 Lov Grover的量子搜索算法 ${ }^{[3]}$ 等已经出现并从理论上证 明可将计算的复杂度由指数增长大大降低到近多项 式增长, 但由于这些算法对量子态操控的容错要求 极高, 从技术上实现真正的量子计算机仍被认为不 可能. 紧接着, 量子纠错算法 ${ }^{[4 \sim 7]}$ 的提出提供了新的 希望一一尽管很渺茫. 很多物理学家认为: 量子计算 机从技术上具备了可行性, 终有一天它会成为现实, 不过那一定是在某个遥远的未来.

然而, 科技的发展总是出人意料. 量子计算近乎 无穷的并行计算能力、量子力学原理更深层的奥秘探 索, 持续地激励世界各国的科研技术人员朝这一“圣 杯” 而努力. 由此展现出来的进步是令人惊叹的, Devoret和 Schoelkopf ${ }^{[8]}$ 在对超导量子电路做综述展
望时说道: “然而, 近20年来我们见证了量子计算、量 子模拟和远距离量子通信等领域如此多的进展，量 子计算机看起来在我们有生之年就能出现.”近几年 来, 随着量子操控精度和读出效率的快速提升, 量子 计算技术的发展已经进人一个新的阶段.一些国际 大型公司与机构, 包括Google, IBM, Intel, Microsoft, Raytheon, NIST, MIT等竞相加人到研发行列, 此外, 一批初创公司, 包括D-wave, IonQ, Quantum Circuits 等不断涌现 (https://quantumcomputingreport.com/), 将量子计算机及量子算法的开发推向新的高度.

量子计算利用了量子态的叠加性和纠缠特性. 叠加性使得量子态的初态可以制备在Bloch球面上的 任意一点, 相比于经典比特只能处在 0 和 1, 初态可包 含更为丰富的信息; 纠缠性则使得量子态所处的态 空间(希尔伯特空间)随比特数增长而指数增长, 极大 地提高了计算的并行能力 ${ }^{[9,10]}$. 通过构造合理的量子 算法, 可以将经典计算机几乎不可能完成的某些 NP 难题, 诸如大数分解、复杂路径搜索等, 在可接受时 
间内解决. 而这些难题的解决将对密码学、大数据和 机器学习、人工智能等信息领域造成深远影响.

量子计算的基本单元称之为量子比特(quantum bit, Qubit). 理论上说, 任何二能级/准二能级系统都 可构成Qubit, 比如自旋为 $1 / 2$ 的电子或原子核、原子 的基态与第一激发态、正交极化的两个单光子态等. 不过, 用于构造技术可行的量子计算机, 对Qubit有 一些最基本的要求. 2000年, 由IBM的DiVincenzo ${ }^{[11]}$ 总结为几条基本准则:

(1) 由具有良好特性的Qubit组成的可扩展物理 系统. “良好特性”意味着对Qubit物理参数的准备掌 握, 包括其内部哈密顿量、与其他Qubit或量子系统的 耦合、与外部场的耦合、与邻近能级的隔离度等. 可 扩展性则是实现量子计算高并行能力的基础.

（2）能够将Qubit制备到某个基准态. 进行量子 计算之前, 必须能够将所有的比特初始化到一个准 确的初态上, 因此每个Qubit必须能够被有效地制备 到某一基准态(例如基态)上. 另一个重要的原因 是, 进行量子纠错需要不断地提供低熵态 Qubits(比 如0态).

(3) 足够长的退相干时间 (远大于单个逻辑门操 作所需时间). 退相干时间描述了Qubit与其周围环境 的接触强度, 或者说, 它描述了Qubit从纯态(量子态) 转变为混态(经典态) 所需的时间. 退相干时间既不能 太短一一量子行为迅速消失, 也不能太长一一量子计 算效率降低. 为了成功地进行量子纠错, 退相干时间 是单个逻辑门操作周期的 $10^{4} \sim 10^{5}$ 倍.

(4) 能够实现一组完备的通用量子门操作. 一个 有效的量子算法一般是由一组么正变换 $U_{1}, U_{2}$, $U_{3} \cdots$ 组成, 每一个变换作用在态空间的某一子空间 上. 对于某一特定的物理系统, 能够实现的变换数量 往往非常有限, 特别是对 3 个以上Qubits进行变换. 所幸的是这些多比特门总可以转换为一系列单比特 门和两比特门的组合. 能够实现一组完备的量子门 操作保证了能够对态空间任一子空间中的Qubits进 行操作.

(5) 能够对任一Qubit进行测量. 在一系列量子 门操作之后, 最终的量子态必须能够被很好地读出. 对任一个Qubit, 需要准确地得到其 0 态和 1 态的占据 率而不影响其他Qubits.

上述这些“准人”规则使得可用作构造实用量子 计算机的候选物理系统非常有限. 研究较多的系统
包括离子阱系统、分子核磁共振系统、量子点、腔量 子电动力学系统、单光子系统、超导宏观量子态等. 在这些候选方案中, 基于超导宏观量子态的超导量 子比特系统由于其易于扩展、易于耦合与操控等特 点, 被认为是最有可能实现容错量子计算机的方案 之一 ${ }^{[12]}$.

\section{1 约瑟夫森结与超导量子比特}

超导量子比特一般由无损耗的电路元件, 包括 电容、电感和约瑟夫森结组成, 一个典型的电路结构 如图1(c)所示 ${ }^{[8,13]}$. 通过调整各电路参数的相对大小, 可以设计出不同的哈密顿量, 从而形成不同的能级 结构. 超导量子比特也常常被称为 “电路量子比特” 或“超导人工原子”. 与自然原子或其他微观量子体 系相比，超导“人工原子”有几个重要的特点. 首先, 它的哈密顿量可以很容易地通过改变电路参数而进 行设计, 具有很大的灵活性; 其次, 它的量子态是由 大量凝聚在基态的库伯对集体表现出来的宏观量子
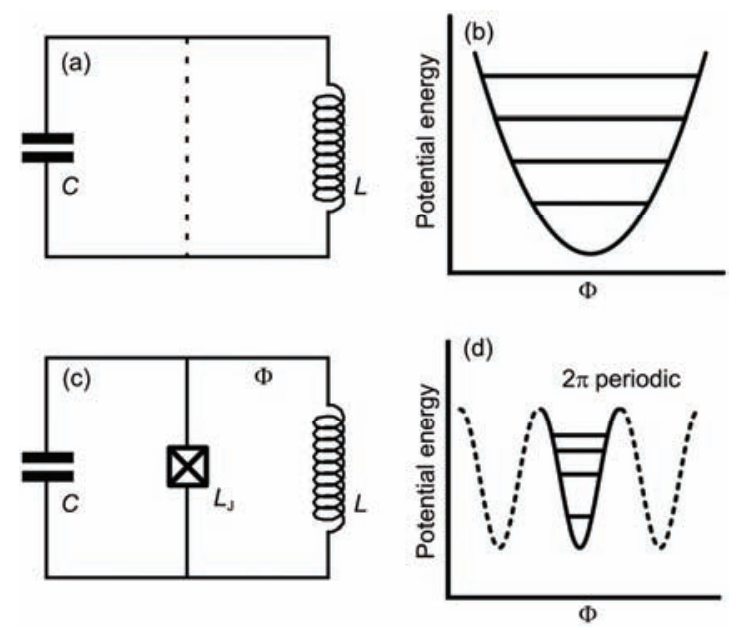

图 1 超导量子电路及其能级结构. (a) 一个简单的线性谐振子电路 模型; (b) 为其能级结构. 对于线性谐振子而言, 所有的能级差是相 同的; (c) 在线性谐振电路中插人一个约瑟夫森结; (d) 为其能级结 构. 由于约瑟夫森结相当于一个非线性电感, 形成的能级结构不是 等间距的. 利用最低两个能级(基态和第一激发态), 可构造一个准二 能级系统, 作为量子计算的基本单元(本图根据文献 $[8,13]$ 改编)

Figure 1 Schematics of superconducting quantum circuits and energy structures. (a) A simple model of linear LC resonator circuit; (b) the energy levels of circuit (a); (c) a Josephson junction is inserted in circuit (a), forming a general model for superconducting qubit; (d) the energy levels of circuit (c). The energy levels become non-equally spaced because of the non-linearity of Josephson inductance. As a result, the lowest two levels (ground state and the first excited state) can be used as a semi two-level system for quantum computing (figures are modified based on Refs. [8,13]) 
态，因此很容易与控制/读出电路进行耦合. 第三, 其第一激发态的能量一般远小于自然原子. 通过合 理的设计, 一般选择在微波(1 10 GHz) 能量范围内, 以利用现有成熟的微波技术对量子态进行操控和读 出. 此外, 构成超导量子比特的电路元件都可以通过 微纳加工手段制备并很好地集成在平面电路中, 因 此与现有成熟的半导体工艺保持了很好的兼容性, 具有良好的扩展性. 从工程技术角度来看, 这些特性 使得超导量子比特相比其他体系而言具有显著的优 势 ${ }^{[13]}$.

然而, 超导量子比特也有其不足之处. 如上所 述, 超导量子态是固态器件中的宏观量子态, 尽管易 于操控和读出, 但与之相伴的是其量子态也易于与 环境发生耦合, 导致相干性快速退化. 最早的超导量 子比特寿命只有不到 $10 \mathrm{~ns}$ 的水平 ${ }^{[14]}$, 尽管能够看到 相干演化, 但难以进行准确地操控. 如何在介观尺度 固态器件复杂的电磁环境中抑制各种噪声影响, 提 高退相干特性成为早期超导量子电路研究的重点内 容. 值得欣慰的是, 在短短十余年时间内, 研究者们 深人探索了退相干/退相位机制, 并对设计、材料、工 艺和外围电路等多个方面进行了大量的优化和改进. 到目前为止, 超导量子比特的退相干时间已经提高 至10 100 $\mu \mathrm{s}$ 量级, 实现了近 6 个数量级的提升 ${ }^{[8]}$, 而 且, 这方面的研究尚未止步. 现有的水平已经能够在 退相干时间内进行 $10^{4}$ 量级的门操作, 很多人认为已 经达到了量子纠错的阈值 ${ }^{[15]}$. 配合高保真度量子非 破坏测量技术的发展, 超导量子计算方案走到了构 建容错量子逻辑比特的阶段. 在整个通用量子计算 技术发展的大蓝图中, 超导方案走在了最前列, 成为 各国政府、大型科技公司和顶尖科研机构竞相角逐的 热门研究领域.

\section{1 非线性电感}

超导量子比特中的关键器件是约瑟夫森结. 电 容和电感可构造LC谐振电路(图 1(a)), 其能级结构是 典型的简谐振子系统 (图1(b)). 尽管其能级是量子化 的, 但简谐振子无法用于构造量子计算机, 因为它所 有的能级差都是一样的, 无法保证在某两个确定的 状态上(比如基态和第一激发态)进行操作. 为了满足 量子比特的要求, 必须在电路中引人一定的非线性. 超导约瑟夫森结是一种几乎无耗散的非线性器件, 自然成为提供这一非线性的最佳选择.
约瑟夫森结一般由两个超导体(也称为超导电 极)及中间薄势垒层构成, 如图2(a)所示. 势垒层(薄 绝缘层)将两侧超导体波函数分开，使得两侧超导体 有各自独立的相位. 同时, 势垒层又足够薄(一般小 于 $100 \mathrm{~nm}$ ), 以至于两侧超导体保持着弱的连接 性. 当两侧超导体相位不等的情况下, 库伯对在相位 差驱动下, 可以在两侧超导体之间隧穿, 形成干涉效 应一一约瑟夫森效应 ${ }^{[16]}$.

约瑟夫森效应最早由 Josephson ${ }^{[17]}$ 提出, 并很快 得到了实验上的验证 ${ }^{[18]}$. 它包含两个主要的关系

$$
\begin{gathered}
I=I_{\mathrm{c}} \sin (\delta), \\
V=\frac{\Phi_{0}}{2 \pi} \dot{\delta},
\end{gathered}
$$

其中, $I_{\mathrm{c}}$ 为约瑟夫森结的临界电流, $\Phi_{0} \sim 2 \times 10^{-15} \mathrm{~Wb}$, 为单个磁通量子. $\delta$ 为两侧超导体之间的相位差 (下文 简称相位). 引人广义磁通量 $\Phi \equiv \int V(t) \mathrm{d} t$, 对约瑟夫 森第二关系式进行积分, 可以得到 $\Phi$ 与相位 $\delta$ 的对应 关系

$$
\delta=2 \pi \Phi / \Phi_{0},
$$

由此, 约瑟夫森电流和电压关系可以改写为

$$
I=I_{\mathrm{c}} \sin \left(2 \pi \Phi / \Phi_{0}\right), V=\dot{\Phi} .
$$

当 $I<I_{\mathrm{c}}$ 时, 约瑟夫森结处于稳态, 结上存在一个 固定相位差, 而此时结两端电压为零, 因此是无耗散 的. 当相位发生变化时, 可以计算出外电流所做的功
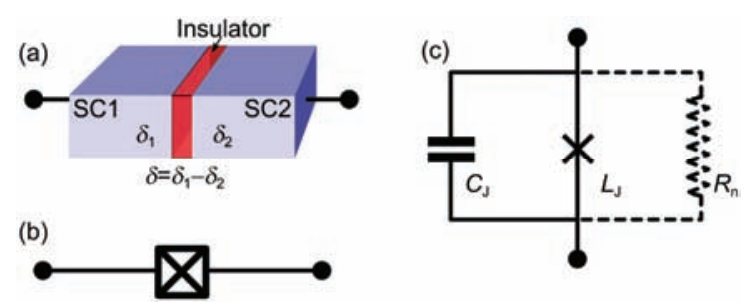

图 2 (网络版彩色)约瑟夫森结基本结构及其等效电路. (a) 一个约 瑟夫森结的典型结构. 它包含左右两个超导体(电极)及中间的薄绝 缘层; (b) 约瑟夫森结在电路中的表示; (c) 约瑟夫森结的电路模型, 它由 3 部分组成: 理想约瑟夫森结、结自身电容和正常态电阻. 当 $I I_{\mathrm{c}}$ 时(在超导量子比特中总是成立的), 正常态电阻 $R_{n}$ 可以忽略, 这里用虚线表示

Figure 2 (Color online) Structure of a Josephson junction and its effective circuit model. (a) Typical structure of a Josephson junction. It consists of two superconductors (electrodes) and a thin insulator layer that separates them; (b) the circuit representation of Josephson junction; (c) a simple effective circuit model for Josephson junction. It consists of three circuit parts: an ideal junction, the self-capacitance of the junction and the normal state resistance 


$$
W_{\mathrm{e}}=\int I V \mathrm{~d} t=\int I_{\mathrm{c}} \sin \delta \frac{\Phi_{0}}{2 \pi} \dot{\delta} \mathrm{d} t=E_{J}\left(\cos \delta_{1}-\cos \delta_{2}\right) .
$$

由于能量没有耗散, 根据能量守恒定律, 这一能 量将存储在结中. 因此, 可以定义一个结的势能 $U(\Phi)$

$$
U(\Phi)=E_{\mathrm{J}}\left[1-\cos \left(2 \pi \Phi / \Phi_{0}\right)\right],
$$

其中, $E_{\mathrm{J}} \equiv \Phi_{0} I_{\mathrm{c}} / 2 \pi$, 称之为约瑟夫森结的特征能量 或约瑟夫森能. 在电路模型中, 这一储能关系可以等 价为一个电感 $L_{\mathrm{J}}$. 根据关系 $V=L \dot{I}$, 可以得到

$$
L_{\mathrm{J}}=\frac{V}{\dot{I}}=\frac{\Phi_{0}}{2 \pi I_{c} \dot{\Phi} \cos \left(2 \pi \Phi / \Phi_{0}\right) / \Phi_{0}}=\frac{L_{\mathrm{J}_{0}}}{\cos \delta},
$$

这里 $L_{\mathrm{J}_{0}}=\Phi_{0} / 2 \pi I_{\mathrm{c}}$, 称之为约瑟夫森结的特征电感. 显然, $L_{\mathrm{J}}$ 是非线性的, 它随相位/电流而改变. 将约瑟 夫森结插人到电路中, 就成为了最基本的超导量子 比特电路(图 1(c)). 图1(d) 则为其势能曲线和能级结 构 (这里假设 $L \rightarrow \infty$, 对应Transmon的情况, 下文将 做详细介绍). 可以看到, 能级结构不再是等间距的, 这时, 可以通过与最低两个能级(基态和第一激发态, 分别作为量子比特的 $\mid 0$ 态和 $\mid 1$ 态)共振的脉冲来制备 并操控量子态.

\section{2 电路参数}

不同的参数选择下, 量子比特的能级结构会发 生很大的变化. 这里进一步引人电容和电感的特征 能量 $E_{C}=e^{2} / 2 C, E_{L}=\left(\Phi_{0} / 2 \pi\right)^{2} / L$, 与约瑟夫森能 $E_{\mathrm{J}}$ 一起, 3 种能量尺度的比例决定了量子比特的构型 (图3).

在超导量子比特发展早期, 出现了几种不同类 型的比特结构. 根据各自量子态的形式分别称为电 荷量子比特 (charge qubit, 也常被称为 copper-pair box)、磁通量子比特(flux qubit)和相位量子比特(phase qubit). 从图3可以看到, 3 种量子比特电路参数差别非 常大, 除电荷量子比特及其衍生的Quantronium 和 Transmon以外, 大部分比特结构还引人了一个额外 的磁通自由度. 量子态不同决定了各种比特结构的 控制和读出方式也有很大的差别. 关于上述 3 种经典 的超导量子比特类型, 国内外有较多的综述文献可 供参考 ${ }^{[12,19 ~ 22]}$, 因此这里不再做赘述. 随着研究者们 对退相干机制了解的逐渐深人, 一些新型的量子比 特结构逐渐出现, 特别是由电荷量子比特发展而来 的 Transmon/Xmon, 由于其结构简单、退相干时间长、

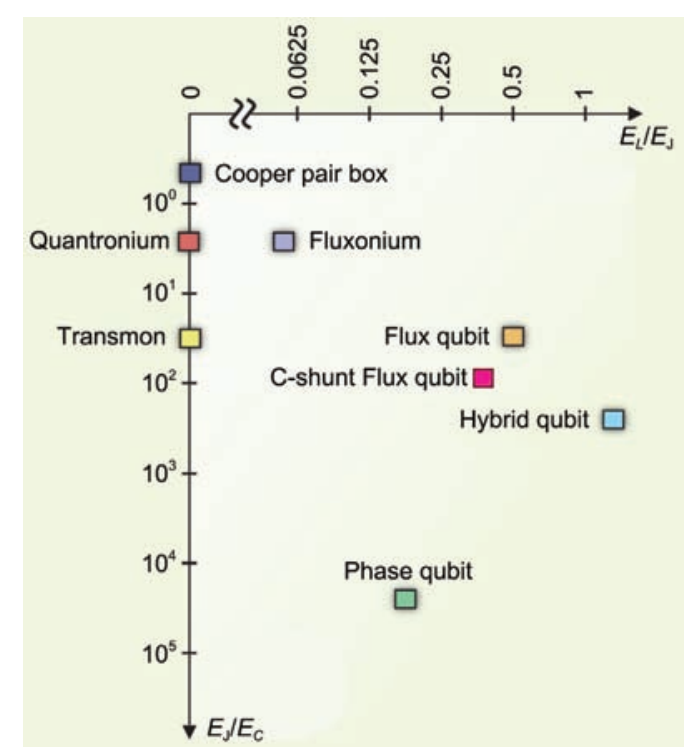

图 3 (网络版彩色)不同类型超导量子比特参数分布示意图(根据文 献[8]修改)

Figure 3 (Color online) A 2D parameter "table" of superconducting artificial atom types (modified based on Ref. [8])

易于操控和读出等优点, 得到了非常广泛的应用. 因 此, 在这里我们将对几种流行的新型量子比特结构 进行介绍, 整理其设计思路, 以期为未来量子比特设 计及架构发展提供些许依据.

\section{2 长退相干时间超导量子比特}

\section{1 如何提高退相干时间}

提高量子比特的退相干时间, 一般有两种思路. 一种是探索材料生长和微纳加工工艺中引人的体/界 面缺陷、杂质等对量子比特的影响, 以此优化材料和 工艺来去除 $1 / f$ 噪声影响. 由于 $1 / f$ 噪声的起源到目 前为止认识并不清楚, 这方面的研究成本很高, 需要 进行大量的摸索和基本测量, 工作量很大. 不过, 由 于这些噪声来源是普适的, 一旦有进展, 对各种量子 比特设计与制备都能带来好处. 举例来说, 相比 $\mathrm{Nb} / \mathrm{AlO}_{x} / \mathrm{Nb}$ 结工艺而言, $\mathrm{Al} / \mathrm{AlO}_{x} / \mathrm{Al}$ 具有更简单的制 备工艺和界面, 可大大降低结区的二能级系统(twolevel system, TLS)缺陷密度, 显著提高退相干特性, 因此全 $\mathrm{Al}$ 结很快在各种量子比特设计中得到广泛应 用; 此外, 关于衬底的介电损耗研究表明, 蓝宝石衬 底具有非常低的介电损耗, 以蓝宝石为衬底制备器 件能够大大改善器件性能 ${ }^{[23]}$ 等.

另一种思路则是设法降低系统对噪声的敏感程 
度. 如果能够找出系统对哪种外界涨落最为敏感, 并 且弄清楚系统对这一涨落的依赖关系, 那么就可以 设法使系统工作在所谓的“甜点 (sweet point)”, 在那 里, 系统能态对涨落的一阶依赖为零 ${ }^{[24]}$. 举例来说, 研究者发现, 影响电荷量子比特退相干时间的主要 因素是电荷涨落. 那么降低这一影响最直接的办法 就是将系统偏置在电荷简并点 $n_{\mathrm{g}}=1 / 2$ 处, 在这里 0 态 和 1 态对应的平均电荷 $\hat{N}$ 都是 $1 / 2$, 因而对门电荷的 一阶涨落不敏感.

\subsection{Transmon/Xmon}

Transmon就是沿着第二种思路向前更进一步发 展出来的, 其全称为“transmission line shunted plasma oscillation qubit”, 最早由Yale大学物理与应用物理 系 Schoelkopf研究组的Koch等人 ${ }^{[25]}$ 于 2007 年提出, 图 4 给出了典型的 Transmon 结构及其电路模型. Transmon的主要思想是, 与其将charge qubit偏置在 门电荷 $n_{\mathrm{g}}=1 / 2$ 处, 不如将系统能态对 $n_{\mathrm{g}}$ 的色散关系平 坦化, 这样处处都是“甜点”. 怎么做到呢? Transmon 的方案是增大约瑟夫森能 $E_{\mathrm{J}}$ 和电荷能 $E_{C}$ 之间的比值 $E_{\mathrm{J}} / E_{C}$. 在电荷量子比特中, 能级随电荷的变化幅度 随 $E_{\mathrm{J}} / E_{C}$ 增加而指数降低, 而非线性随 $E_{\mathrm{J}} / E_{C}$ 则是幂指 数降低. 因此, 适当降低 $E_{\mathrm{J}} / E_{C}$, 可极大地降低系统对 电荷的敏感度，同时仍能保留足够的非线性. 图5显 示了不同 $E_{\mathrm{J}} / E_{C}$ 下量子比特能级随电荷的变化关系. 可以看到, 当 $E_{\mathrm{J}} / E_{C}$ 达到 50 时, 能级随门电荷 $n_{\mathrm{g}}$ 的变 化已经完全趋于平坦, 相应的能级结构逐渐趋于简 谐振子形式.

从图4的电路结构上看, 实际上Transmon就是在 约瑟夫森结两端并联一个较大的电容, 此外, Trans$\operatorname{mon}$ 的门电容 $C_{\mathrm{g}}$ 改为与一个线性谐振腔之间的耦合 电容, 这一耦合使得量子比特与谐振腔构成一个电 路量子电动力学(circuit-QED)系统, 可实现对量子比 特的操控和读出. 电容的增大使得约瑟夫森结两端 超导电极容纳的电荷数大大增加, 此时电荷已经不 是一个好量子数, 系统能态从典型的电荷态转变为 “等离子体 (plasma)态”, 即电荷与相位的振荡态. 如 果将 Transmon中的一个结置换为由两个结组成的 SQUID环，通过外加磁通还可以控制 $E_{\mathrm{J}}$ 的大小，从而 实现能级可调, 其代价是引人了新的退相干因素— 磁通涨落.

Transmon结构一经提出便取得了巨大的成功,

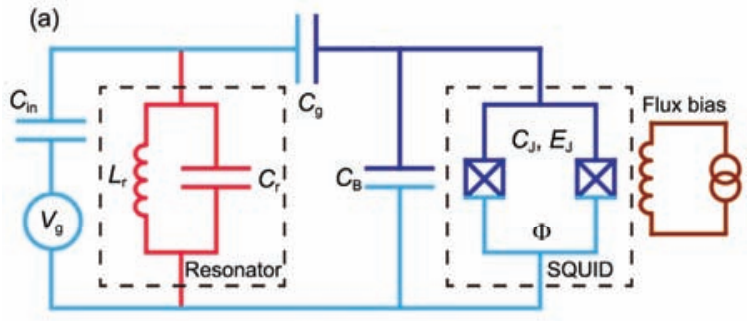

(b)

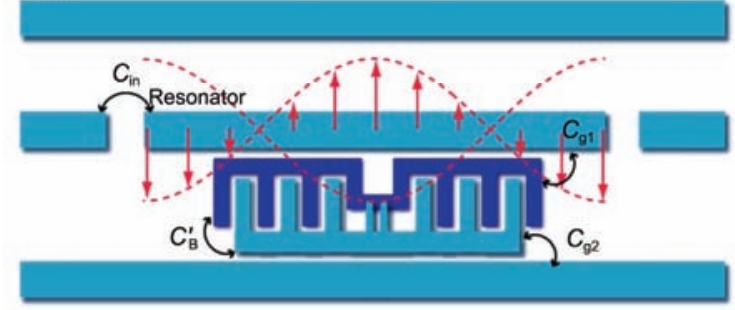

图 4 (网络版彩色)Transmon结构示意图及其等效电路图 ${ }^{[25]}$. (a) Transmon的等效电路模型. $V_{\mathrm{g}}$ 为门电压, 通过 $C_{\mathrm{in}}$ 耦合到谐振腔, 再通 过 $C_{\mathrm{g}}$ 与量子比特耦合. 量子比特为一个约瑟夫森结或由两个结及小 的超导回路组成的SQUID环路构成, $C_{\mathrm{B}}$ 提供了一个大的旁路电容. SQUID环路磁通可由外部电感耦合产生, 通过这一磁通 $\Phi$ 可调节等 效的 $E_{\mathrm{J}}$ 大小, 进而调节 $E_{\mathrm{J}} / E_{\mathrm{C}}$; (b) Transmon的平面设计结构. 一个小 的SQUID (提供 $E_{\mathrm{J}}$ )与叉指电容的两个极板相连 (提供 $C_{\mathrm{B}}$ ), 然后放置在 一个 $\lambda / 2$ 谐振腔中心, 与谐振腔的耦合电容 $\left(C_{\mathrm{g} 1}\right.$ 和 $\left.C_{\mathrm{g} 2}\right)$ 提供 $C_{\mathrm{g}}, C_{\mathrm{in}}$ 则是 谐振腔与外部传输线之间的耦合电容

Figure 4 (Color online) Schematic of a transmon qubit and its effective circuit ${ }^{[25]}$. (a) The effective circuit model of a transmon qubit. Here $V_{\mathrm{g}}$ is the gate voltage, which is coupled to the resonator by $C_{\mathrm{in}}$, and is then coupled to the qubit by $C_{\mathrm{g}}$. A transmon qubit is a Josephson junction or a SQUID loop shunted with a large capacitor $C_{\mathrm{B}}$. External flux $\Phi$ can be used to tune the effective Josephson energy $E_{\mathrm{J}}$, so as $E_{\mathrm{J}} / E_{C}$; (b) a schematic of $2 \mathrm{D}$ structure of a transmon qubit. A small SQUID loop is connected with the two arms of an interdigital capacitor, which is laid in the gap of a $\lambda / 2$ coplanar waveguide transmission line resonator

其退相干时间迅速提高到 $10 \mu \mathrm{s}$ 量级，吸引了很多研 究组的加人, 并很快衍生出几种典型的变异结构. 其 中最具代表性的包括UCSB Martinis研究组的Xmon结 构 ${ }^{[15,26]}$ 和Yale大学 Schoelkopf研究组的3D-transmon结 构 ${ }^{[27]}$.

Xmon的设计基于平面电路, 如图6所示. 其主要 的设计思想包括:

(1) Transmon中两个电容电极之一由共同的地取 代，另一电极则设计为十字结构，便于与邻近其他量 子比特及谐振腔进行耦合.

(2) Xmon与一个 $\lambda / 4$ 谐振腔的开放端通过爪式电 容耦合, 谐振腔则与一个共同的读出传输线 (readout line)耦合. 在这里谐振腔只用于读出, 而不再用于量 子态操控. 

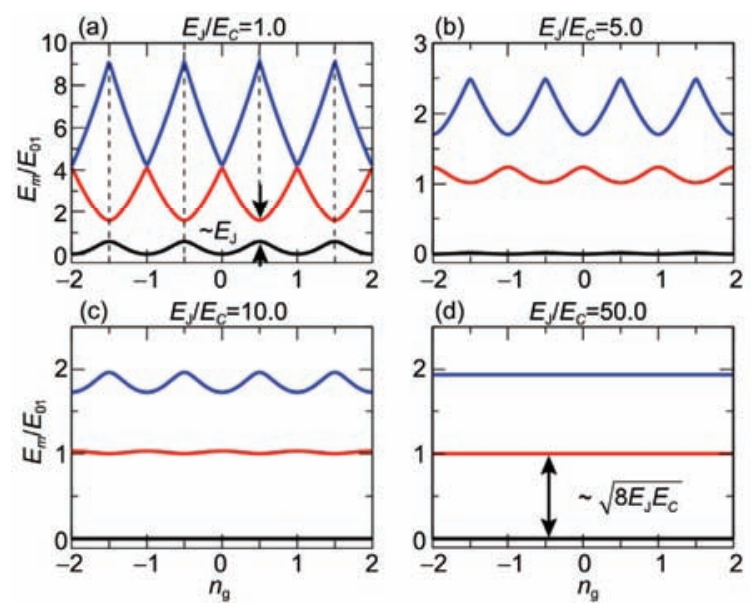

图 5 (网络版彩色) Transmon哈密顿量的最低 3 个能级随门电荷 $n_{\mathrm{g}}$ 的 变化关系 ${ }^{[25]}$. (a) $E_{\mathrm{J}} / E_{C}=1.0$; (b) $E_{\mathrm{J}} / E_{C}=5.0$; (c) $E_{\mathrm{J}} / E_{C}=10.0$; (d) $E_{\mathrm{J}} / E_{C}=$ 50.0. 随着 $E_{\mathrm{J}} / E_{C}$ 逐渐增大, 能级随 $n_{\mathrm{g}}$ 的变化迅速趋于平坦化, 同时能 级非线性也相应的降低. 当 $E_{\mathrm{J}} / E_{C}$ 时, 能级结构趋向于简谐振子

Figure 5 (Color online) The lowest three levels of a transmon qubit as a function of the effective offset charge $n_{\mathrm{g}}{ }^{[25]}$. (a) $E_{\mathrm{J}} / E_{C}=1.0$; (b) $E_{\mathrm{J}} / E_{C}=5.0 ;$ (c) $E_{\mathrm{J}} / E_{C}=10.0 ;$ (d) $E_{\mathrm{J}} / E_{C}=50.0$. As $E_{\mathrm{J}} / E_{C}$ increases, the eigenenergies become flat, in the meantime, the anharmonicity is decreased. When $E_{\mathrm{J}} / E_{C}$, the eigenenergies approach to that of a harmonic oscillator

(3) 每个Xmon通过两根独立的控制线进行操控, 其中一根与 $X m o n$ 电容耦合, 可实现量子态 $X$ 或 $Y$ 方向 的旋转操作, 称之为 $X / Y$ 控制线, 另一根与 $X$ mon电感 耦合, 可实现Xmon的能级差调节和 $Z$ 方向旋转操作, 称之为 $Z$ 控制线.

Xmon设计架构非常优雅. 它能够很好地实现所 有量子比特的完全控制 $(X, Y, Z$ 独立旋转), 同时又能 够实现所有量子比特的同时读出 (在后级读出放大电 路带宽足够的情况下), 各比特之间通过电容直接耦 合, 这种近邻耦合结构刚好能够满足表面编码 (surface code) 量子纠错的需求, 适合于进行大规模扩 展. 2014年, Barends等人 ${ }^{[15]}$ 报道了在一个 5 比特系统 中的量子门操作随机基准测试(random benchmarking), 对Clifford群(一组完备的量子门操作群)中所有 的单比特门和双比特门操作保真度标定, 结果表明 所有单比特门操作保真度均 $>99.9 \%$, 双比特 CZ门操 作保真度 $>99 \%$. 这一结果被认为已经达到了表面编 码量子纠错的阈值要求.

在后续的设计中, Xmon架构进一步增加了 "Purcell filter" ${ }^{, 28]}$, 这一设计将读出的传输线更换为 一个低 $Q(Q \sim 30)$ 的谐振腔, 并控制谐振腔的带宽正好 能够覆盖所有读出谐振腔的频率范围 $(\sim 300 \mathrm{HMz})$.

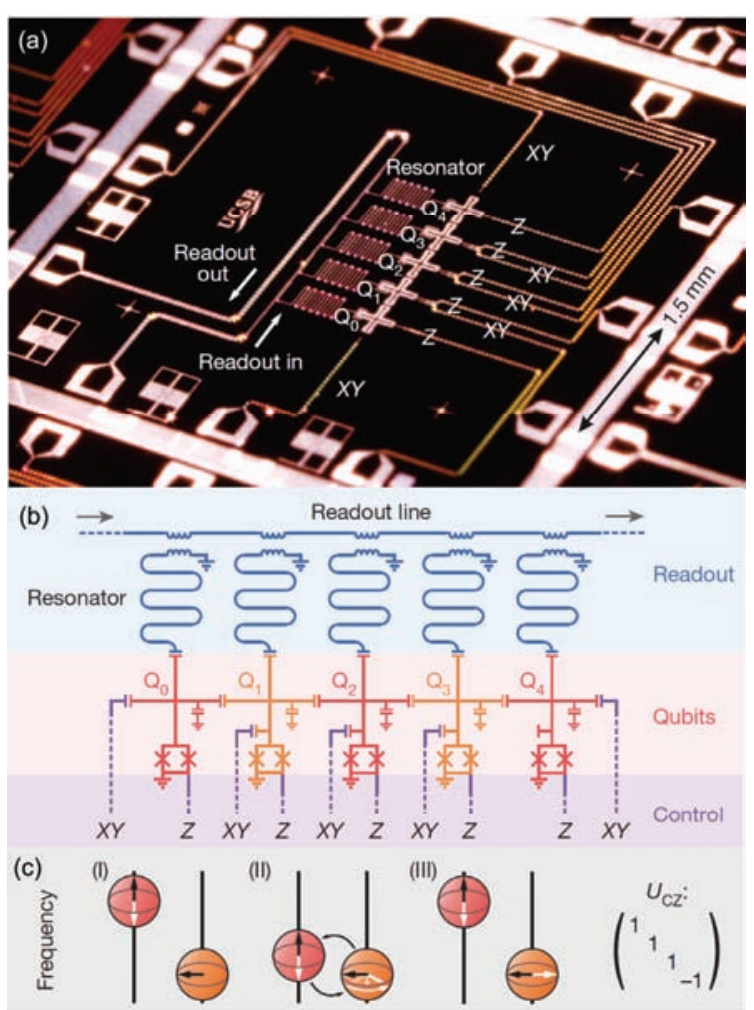

图 6 (网络版彩色)多比特Xmon系统 ${ }^{[15]}$. (a) 一个包含 5 个量子比特 的Xmon结构. $\mathrm{Q}_{0} \sim \mathrm{Q}_{4}$ 为Xmon量子比特, 每个比特通过电容与一个读 出谐振腔耦合. 所有的读出谐振腔与一个宽带的传输线(readout line) 耦合. Xmon的旁路电容为一个十字形的电极, 能够与旁边的比特通 过电容发生近邻耦合. (b) 多比特Xmon系统的电路模型. 每个比特 拥有两根独立的控制线 $(X Y$ 和 $Z)$, 其中 $X Y$ 线与十字形电极通过电容耦 合, 用于 $X$ 和 $Y$ 方向的旋转操作, $Z$ 线与SQUID环路通过互感耦合, 用 于调整量子比特能级差及 $Z$ 方向旋转操作

Figure 6 (Color online) A multi-qubit Xmon system ${ }^{[15]}$. (a) A micrograph of a 5-bit Xmon system. $\mathrm{Q}_{0}-\mathrm{Q}_{4}$ are Xmon qubit units. Each of them is coupled to a readout resonator by a claw like capacitor. All the readout resonators are then coupled to a wideband transmission line (readout line). The shunted capacitor of an Xmon is a cross like electrode, so it can directly couple to its nearest qubits by capacitors. (b) An effective circuit for device in (a). Each qubit has two independent control line $(X Y$ and $Z$ ). The $X Y$ line, which is coupled to the qubit by a small capacitor, is used to do $X$ and $Y$ rotations. The $Z$ line, which is coupled to the SQUID loop of the qubit, is used to tune the energy levels and do $Z$ rotation

Purcell filter的主要作用在于，它能够进一步压制由 于Purcell效应造成的退相干，同时进一步提高读出 速度. 2015年, Kelly等人 ${ }^{[29]}$ 在一个9比特系统中演示 了一维表面编码纠错, 对 GHZ态进行重复编码测量, 经过 8 个测量周期之后, 错误率降低了 8.5 倍, 表明了 表面编码量子纠错的有效性.

在这里值得一提的是，最近几年国内在超导量 子比特器件制备和测量方面发展非常快. 例如, 在中 
国科学院物理研究所制备的 10 比特和 5 比特的样品已 经用于浙江大学王浩华小组和包括中国科学技术大 学、中国科学院物理研究所、福州大学、杭州师范大 学以及美国堪萨斯大学等合作进行的多比特量子纠 缠的实验 ${ }^{[30]}$, 其中 10 比特的Xmon器件上实现了全纠 缠 GHZ态, 保真度达到 $0.668 \pm 0.025$. 这是到目前为 止在固态器件中实现的最大量子纠缠. 这些样品的 退相干时间可以达到 $20 \mu \mathrm{s}$ 以上. 最新的测量结果显 示, 最好的退相干时间可以达到 $40 \mu \mathrm{s}$. 当然, 整体上 讲, 国内在实验技术研究方面与国外最高水平还是 有明显差距的.

3D-transmon是将Transmon放置在一个由封闭金 属空腔构成的谐振腔(3D-谐振腔)内, 如图7所示. 在 极低温下, $3 \mathrm{D}$-腔由于其电磁场几乎完全被约束在真 空内, 因而可获得极高的 $Q$ 值 $\left(10^{6} \sim 10^{7}\right.$ 量级). 约瑟夫 森结两端电容电极与 $3 \mathrm{D}$-腔中的微波场耦合, 3D-腔 则通过两个输入输出端口与外电路耦合, 等效电路 与图 4(a)一样. 此外, 在3D-transmon中, 旁路电容变 为两个相近的大面积电极板, 与 3D-腔的耦合电容介 质层相当于是真空, 而真空的介电损耗趋近于零, 因 此可极大地提高量子比特的寿命.

3D-transmon结构是一个极佳的电路量子电动力 学系统, 其良好的退相干性能能够进行一些量子算 法演示或用于研究一些量子力学基本问题 ${ }^{[31,32]}$. 3D腔提供的良好电磁环境是关键. 不过, 长远来看, 3D-transmon在向实用量子计算技术发展中面临的一 个主要困难是扩展性不如Xmon, 尽管Yale大学研究 组随后提出 “2.5D电路”的概念 ${ }^{[33]}$, 试图将 $3 \mathrm{D}$-腔微型 化并与平面器件集成, 但表现仍不尽人意, 工艺和设

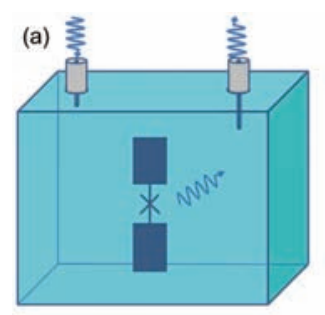

(b)

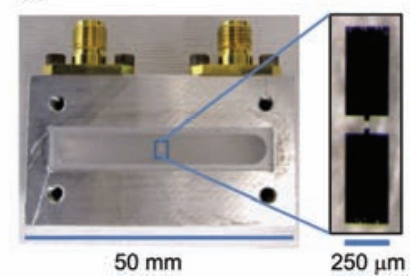

图 7 (网络版彩色)3D-Transmon系统 ${ }^{[27]}$. (a) Transmon与 3D-腔构成 的量子电动力学系统; (b) 3D-Transmon实物图. Transmon(最右放大 图)位于 3D-腔的中心位置(电场最强处)

Figure 7 (Color online) 3D-Transmon system ${ }^{[27]}$. (a) A schematic of a circuit-QED system with a transmon qubit coupled to a 3D-cavity; (b) a picture of a 3D-transmon sample. The Transmon qubit (enlarged in the right) was placed in the center (where the electric field is at maximum) of a 3D-cavity
计上都有待进一步的提升.

\subsection{Fluxonium}

Fluxonium $^{[34]}$ 是另一种非常值得关注的新型超导 量子比特(图 8). 与 Transmon同样, 它也试图解决电 荷量子比特中电荷涨落造成的退相干问题，但采用 了完全不同的办法. 在电荷量子比特中, 为了保证电 荷是一个好量子数(即电荷分布的量子涨落应小于一 个库伯对电荷), 要求结面积足够小, 用电路的语言 来说, 即要求 $Z_{\mathrm{J}}>R_{\mathrm{Q}}$, 这里 $R_{\mathrm{Q}}$ 是超导量子阻抗: $R_{\mathrm{Q}}=\hbar /(2 e)^{2} \sim 1 \mathrm{k} \Omega$. 由于小的超导岛上总电荷数少, 在固态器件的电荷环境中, 很容易受到电荷漂移的 影响, 这正是电荷量子比特面临的困境. 降低这一影 响的直接办法是采用大结, $Z_{\mathrm{J}}>R_{\mathrm{Q}}$, 这时总电荷数大 大增加, 漂移电荷的影响就被抑制了, 但这与电荷量 子比特的要求却是矛盾的. Fluxonium的解决办法是, 将很多的大结串联起来, 然后与小的电容(小结)并 联. 整个回路中不存在小的超导岛, 避免了电荷漂移 的影响, 同时, 大结串联提供了足够大的电感, 因此 仍然能够保证 $Z_{\mathrm{A}}>R_{\mathrm{Q}}$ 的要求, 系统的量子态仍能保持 为电荷态.

当系统振荡频率远低于大结的等离子振荡频率 时, 大结串联阵列的动力学行为相当于一个大的线 性电感 $L_{\mathrm{A}}$. 从电路的角度来看, 这样的大电感相当于 一个“积分器”, 或者说低通滤波器. 因此, 小电容两 端电荷的低频变化被这一大电感短路掉了, 而高频 振荡则不受影响. 这样一来, Fluxonium很好地抑制 了低频电荷漂移, 同时又保留了电荷的高频振荡部 分. 与 Transmon增加电容, 牺牲非线性来抑制电荷 噪声相比, Fluxonium几乎保持了电荷量子比特的各 种特性, 包括高非线性. 此外, Fluxonium增加了一个 新的自由度一环路磁通 $\Phi_{\mathrm{ext}}$. 通过改变 $\Phi_{\mathrm{ext}}$, 其能级 结构能够在很大范围 $(0.5 \sim 10 \mathrm{GHz})$ 内可调. 这一新 增自由度可用于研究很多新的问题, 比如接下来介 绍的准粒子隧穿干涉问题, 以及可用于构造 “ $\Lambda$ ”或 “V”型能级结构、构造“人工分子”等.

在约瑟夫森效应的研究中, 直流输运及动力学 特性已经非常清楚了, 但高频下的某些特性, 受实验 条件限制, 一直未能得出结论. 其中一个问题是准粒 子在高频下的输运问题. Langenberg ${ }^{[35]}$ 在进一步研究 约瑟夫森效应时, 提出了一个理论预言: 准粒子耗散 在约瑟夫森结相位差为 $\pi$ 时会等于零. 当结上存在相 

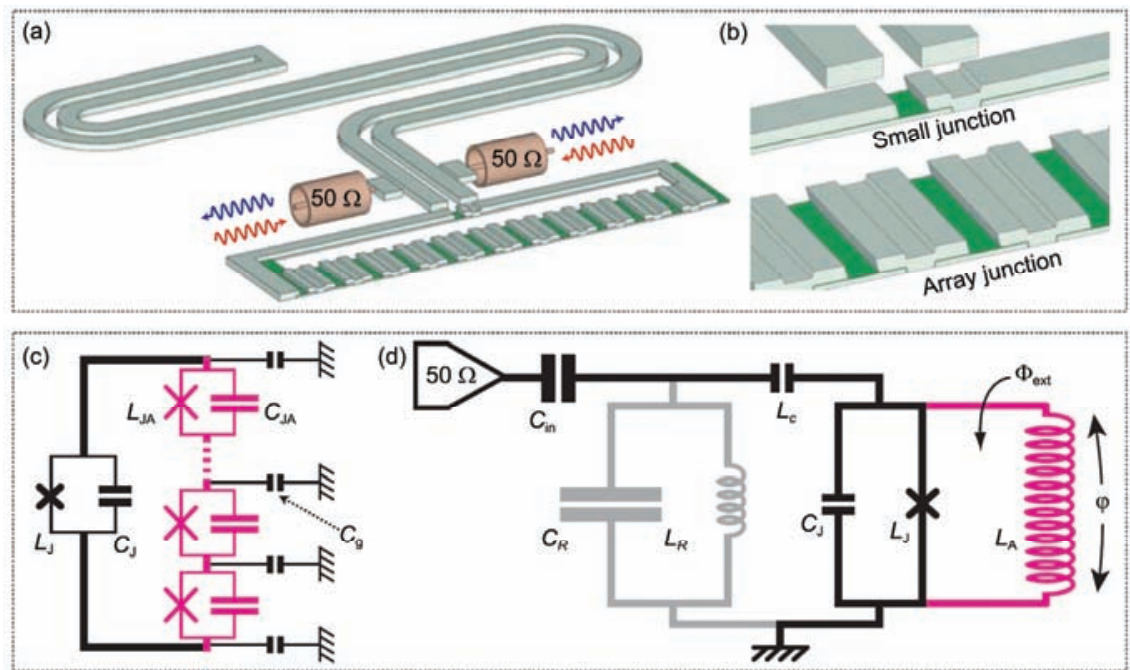

图 8 (网络版彩色)Fluxonium量子比特及其等效电路图 ${ }^{[34]}$. (a) Fluxonium量子比特及其与谐振腔构成的Circuit-QED系统示意图; (b) Fluxonium 放大结构示意图; (c) Fluxonium等效电路; (d) Fluxonium及其Circuit-QED系统的等效电路示意图. 由于大结串联阵列非线性被压制, 可以用一 个大电感 $L_{\mathrm{A}}$ 来表示

Figure 8 (Color online) Fluxonium qubit and its effective circuit model ${ }^{[34]}$. (a) A circuit-QED system with a fluxonium coupled to a coplanar waveguide resonator; (b) the enlarged part of the fluxonium in (a); (c) the effective circuit of a fluxonium qubit; (d) a schematic of the whole circuit-QED circuit of a fluxonium coupled to a resonator. As the nonlinearity is suppressed, the Josephson junction series can be considered as a large linear inductance $L_{\mathrm{A}}$

位差 $\varphi$ 时, 电子型和空穴型准粒子隧穿后将分别获得 相位 $\varphi / 2$ 和 $-\varphi / 2$, 由于这两种隧穿过程不可分, 最终 的隧穿几率为两个过程的相干叠加, 从而产生一个 $(1+\cos \varphi)$ 项: $\left|\mathrm{e}^{\mathrm{i} \varphi / 2}+\mathrm{e}^{-\mathrm{i} \varphi / 2}\right|^{2}=2(1+\cos \varphi)(\text { 图9(a) })^{[36]}$. 很 显然, 当 $\varphi=\pi$ 时, 准粒子隧穿的总几率将变为零.

如上所述, Fluxonium提供了一个新的磁通自由 度, 因而可以用于研究这个问题. Pop等人 ${ }^{[36]}$ 通过系 统研究Fluxonium在不同磁通偏置下的弛豫时间 $T_{1}$, 发现当外加磁通在 $\Phi_{0} / 2$ 附近时, $T_{1}$ 发生了急剧的变化, 在 $0.02 \Phi_{0}$ 范围内, 退相干时间提高了超过一个数量 级, 并在 $\Phi_{0} / 2$ 处达到极值 $8.1 \mathrm{~ms}$. $T_{1}$ 是量子比特能量 耗散的特征时间, 这一结果给出了准粒子隧穿相干 相消的明显证据. 超过 $8 \mathrm{~ms}$ 的退相干时间更是令人 印象深刻. 即便与目前最高水平的其他超导量子比 特相比, 也高出了一个数量级.

实际上, 当 $\Phi_{\mathrm{ext}}=\Phi_{0} / 2$ 时, Fluxonium的行为更接 近磁通量子比特. 它的基态和第一激发态是由势能 底部的双势阱中两个简并基态耦合䢃裂形成的, 如 图9(c)插图所示. 遗憾的是Fluxonium的参数选择是 按照电荷量子比特设计的, 在 $\Phi_{0} / 2$ 处, 它的能级䢃裂 只有不到 $1 \mathrm{GHz}$, 不适合用于量子计算研究. 此外, 尽管此处的能量弛豫时间 $T_{1}$ 很长, 但退相干时间 $T_{2}$ 相 对较短 ( 10 $\mu \mathrm{s}$ 量级), 这与环路中的磁通噪声有密切 (a)
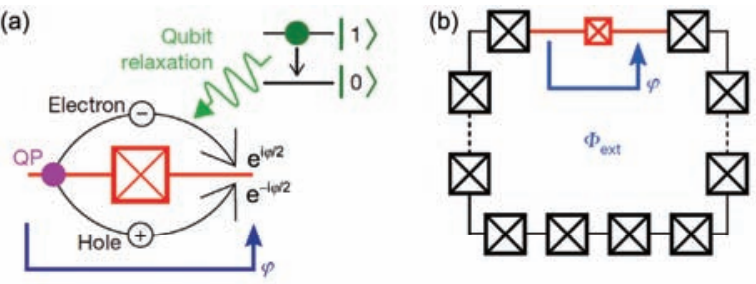

(c)

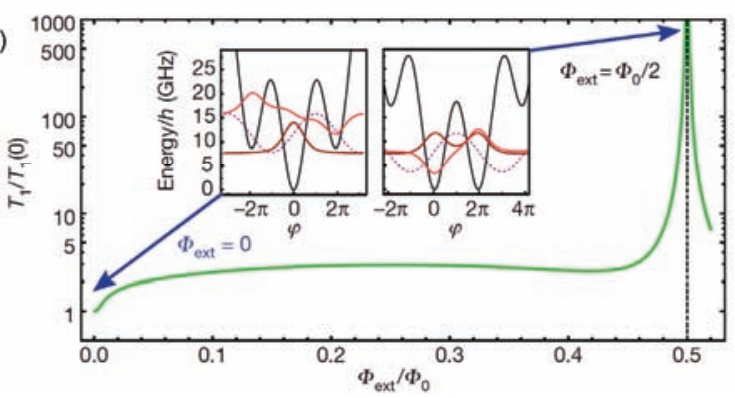

图 9 (网络版彩色)约瑟夫森结准粒子耗散的相位依赖模型 ${ }^{[36]}$. (a) 约瑟夫森结准粒子隧穿干涉过程. 准粒子隧穿过程中会与量子比特 的能态发生相互作用, 导致弛豫, 是量子比特退相干的主要因素之 一; (b) Fluxonium的结构示意图, 它是由一组大结和一个小结串联而 成的环路; (c) Fluxonium能级弛豫时间 $T_{1}$ 随外加磁通的变化. 当 $\Phi_{\mathrm{ext}}=\Phi_{0} / 2$ 时, 由于准粒子隧穿耗散过程被抑制, $T_{1}$ 急剧增加

Figure 9 (Color online) Phase-dependent quasiparticle dissipation in a Josephson junction ${ }^{[36]}$. (a) The interference process of tunneled quasiparticles as electrons or holes, quasiparticle tunneling is an important source of qubit relaxation; (b) a schematic of the fluxonium qubit structure, it consists of a series of large junctions acting as a large inductance, and a small junction; (c) the energy relaxation time $T_{1}$ of a fluxonium, as a function of external flux. When $\Phi_{\text {ext }}=\Phi_{0} / 2$, the quasiparticle tunneling process is suppressed, as a result, $T_{1}$ increases drastically 
的关系, 如何抑制磁通噪声影响将在下节详细讨论.

\subsection{C-shunt flux qubit}

磁通量子比特也是有很长研究历史的一种典型 超导量子比特. 相比于电荷量子比特的发展而言, 其 退相干性能提升的进展要慢得多. 不过, 2016年, Yan 等人 ${ }^{[37]}$ 提出了一种改进型磁通量子比特, 名为 “C-shunt flux qubit (CSFQ)”. 实验结果表明, 这种结 构具有与 Transmon相当的退相干特性, 同时具有高 于Transmon的非线性( 500 MHz). 顾名思义, 这一 新型量子比特在磁通量子比特基础上, 并联了一个 电容. 但变化不仅于此.

在磁通量子比特上并联一个电容的概念其实早 在 2007 年就由我国科学家You等人 ${ }^{[38]}$ 提出了, 发表 的时间甚至早于Koch等人 ${ }^{[25]}$ 提出的 Transmon设计. 比较有趣的是, 2006年Martinis研究组在相位量子比 特研究中, 就提出并验证了通过并联一个“低损耗的 电容”, 可极大地改善量子比特的性能 ${ }^{[39]}$. 可见在当 时, 增大电容来提升量子比特性能已经成为共识.

在You等人的设计中有 3 个主要的思想: (1) 适当 降低 $E_{\mathrm{J}} / E_{C}$ 比率可降低磁通量子比特对磁通噪声的敏 感度, 但电荷噪声的影响将变得更加显著; (2) 适当 降低 $\alpha$ 值可以进一步抑制磁通噪声, 并且显著提高偏 离简并点 $\left(\Phi_{\mathrm{ext}}=\Phi_{0} / 2\right)$ 处的退相干特性; (3) 电荷噪声 可以通过在小结上并联一个电容来有效抑制.
但论文同时也强调了上述几种参数调节的限制 条件, 包括 $\alpha$ 不能小于 0.5 , 否则双势阱结构消失而变 成单势阴, 能级结构将转变为相位型. 另外并联电容 不能过大, 否则大电容中介质层内的缺陷和声子过 程将提供新的退相干来源.

IBM Watson研究中心很快将这一设计思想应用 到器件制备中 ${ }^{[40]}$, 不过他们采用了一个很小的 $\alpha=$ 0.3 , 因此能级结构与 Transmon相似, 但非线性相反 (Transmon的高能级间隔是逐渐减小的, 而这一结构 则是逐渐增大的). 实验结果表明退相干时间有较显 著的提升 $\left(T_{2} \sim 1.5 \mu \mathrm{s}\right)$, 与同时期的 Transmon实验结果 相当. 与此同时, UC Berkeley的John Clarke研究组也 在进行这方面的研究 ${ }^{[41]}$, 但经过大量的摸索, 不断 修改设计之后, 退相干时间仍然在 $1 \mu \mathrm{s}$ 量级, 没有显 著的提升. 而此时 Transmon/Xmon则迅速提升到了 $10 \mu \mathrm{s}$ 以上. 在面临一系列困惑时, Clarke研究组的合 作方 MIT(Massachusetts Institute of Technology) 及 MIT林肯实验室在几乎同样的工艺条件下，得到了 $20 \mu \mathrm{s}$ 以上的退相干时间. 这一令人振奋的结果背后, 到底存在哪些变化呢?

MIT的CSFQ基本结构与IBM和UC Berkeley相似 (图 10), 不过有一些细节上的变化：(1) 将叉指电容 改为大面积的电极块; (2) $\alpha$ 值选择为接近但小于 0.5 ; (3) 电容和地层采用退火的蓝宝石衬底上分子束外 延A1膜制备.
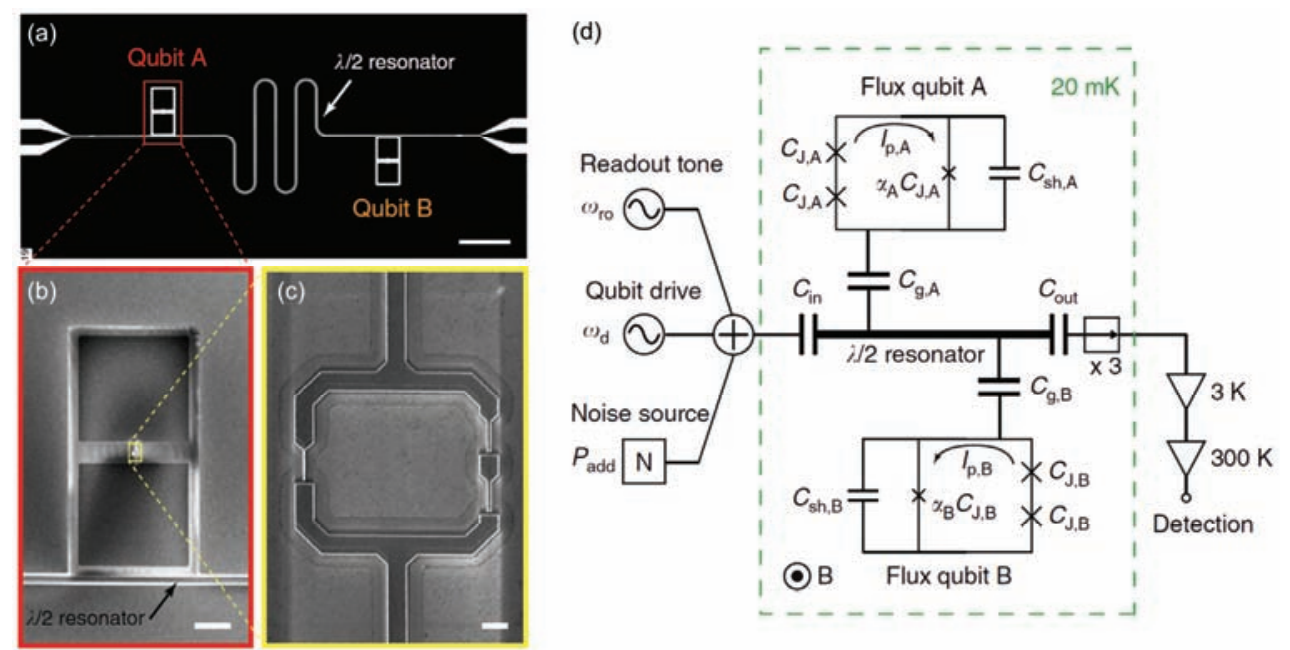

图 10 (网络版彩色)CSFQ及其等效电路图 ${ }^{[37]}$. (a) CSFQ器件照片, 两个量子比特与同一个 $\lambda / 2$ 的CPW谐振腔耦合起来; (b) 量子比特放大照片; (c) 量子比特中心约瑟夫森结环路放大照片; (d) 总的等效电路示意图

Figure 10 (Color online) CSFQ and its effective circuit in a circuit-QED system ${ }^{[37]}$. (a) A micrograph of a CSFQ sample, here 2 qubits are coupled to a $\lambda / 2$ coplanar waveguide resonator; (b) a enlarged picture of a CSFQ; (c) the junctions and the superconducting loop in the center of the CSFQ in (b); (d) the effective circuit model of the structure shown in (a) 
在所有测试过的 22 个 CSFQ样品中, $\alpha$ 值的选取 均接近于 $0.5(0.4 \sim 0.6)$. 结果表明, 所有 $\alpha>0.5$ 的样品, $T_{1}$ 均不超过 $10 \mu \mathrm{s}$, 而所有 $\alpha<0.5$ 的样品, $T_{1}$ 均高于 $20 \mu \mathrm{s}$, 最长超过 $50 \mu \mathrm{s}$. 在 $\alpha<0.5$ 的样品中, 采用叉指型电容 的样品 $T_{1}$ 又显著低于方块形电容样品.

综合分析CSFQ的发展过程, 材料、工艺和设计 等几个因素对退相干特性的改善都非常重要. UC Berkeley与MIT的器件是在相同的工艺条件下做的, 差别应来源于高质量 $\mathrm{Al}$ 膜和更为合理的设计(弃用叉 指电容等). IBM的器件采用 $\mathrm{Si}$ 基片上的 $\mathrm{Nb}$ 膜作为电 极/地层, 可能也是限制退相干时间的重要因素.

\section{3 总结与展望}

上述3种典型的改进型超导量子比特, 显示了研 究者们在提高退相干特性方面做出的卓越努力. 而 从这几种看似差别很大的设计结构中, 依稀可以看 出一些共同的趋势. 可以大致列举如下.

（1）去小岛化, 即增大小结两端超导岛的面积, 这是抑制电荷噪声的最佳办法. 实现这一点最直接 的方式是并联一个旁路电容. 在Fluxonium中, 尽管 电容没有增大, 但将小结与一大串大结串联形成回 路之后，整个回路中小面积的超导岛就没有了.

（2）去环路化或降低环路电流以降低系统对磁 通涨落的敏感度. 在Transmon中, 直接将环路去掉 了, 而在 CSFQ中则是大幅压低 $\alpha$ 和 $E_{\mathrm{J}}$, 甚至连双势阱 结构也去掉了, 环流态已不再是好量子态.

(3) 改变小结的工作相位. 当工作点选取为小结 上相位差为 $\pi$ 时, 准粒子隧穿相干相消, 可大幅抑制 准粒子噪声。

(4) 改善量子比特的电磁环境. 采用电路量子电 动力学结构, 利用高 $Q$ 的线性谐振腔与超导量子比特 色散耦合, 由此大幅降低系统与环境耦合的带宽.

进一步提高超导量子比特的退相干性能和非线 性, 将大幅提升以之为基础的超导量子计算技术方 案的实用性和可行性, 推动超导量子计算走向新的 阶段. 关于这方面的研究远未止步, 我们相信在很短 的未来, 将出现更多新颖的比特单元和架构设计. 与
此同时，材料和工艺、控制与测量、集成与封装等方 面的研究也将进一步优化和深人.

回顾过去几年的发展，退相干时间的提升是呈 指数加速的, Oliver等人 ${ }^{[42]}$ 总结了这一规律, 并称之 为退相干时间的“摩尔定律”，如图11所示. 这一规律 还将持续一段时间, 但受到固态器件复杂的电磁环 境限制, 且随着量子态操作和读出保真度提高到一 定水平, 退相干时间的提升终将放缓. 但超导量子计 算的“摩尔定律”不会终结，随着控制、测量和封装等 技术的进步, 超导量子电路在扩展和集成方面, 将沿 着新的“摩尔定律”继续走下去. 我国在超导量子计 算方面起步较晚, 特别在实验方面, 与国际最高水平 仍有较大差距. 不过, 在国家科技部门的高度重视 下，一批富有才华的青年科学家队伍已经成长起来, 器件制备与测量发展速度非常之快, 令世界瞩目. 相 信在不久的将来, 我国科学家将在超导量子计算技 术发展中扮演重要角色.

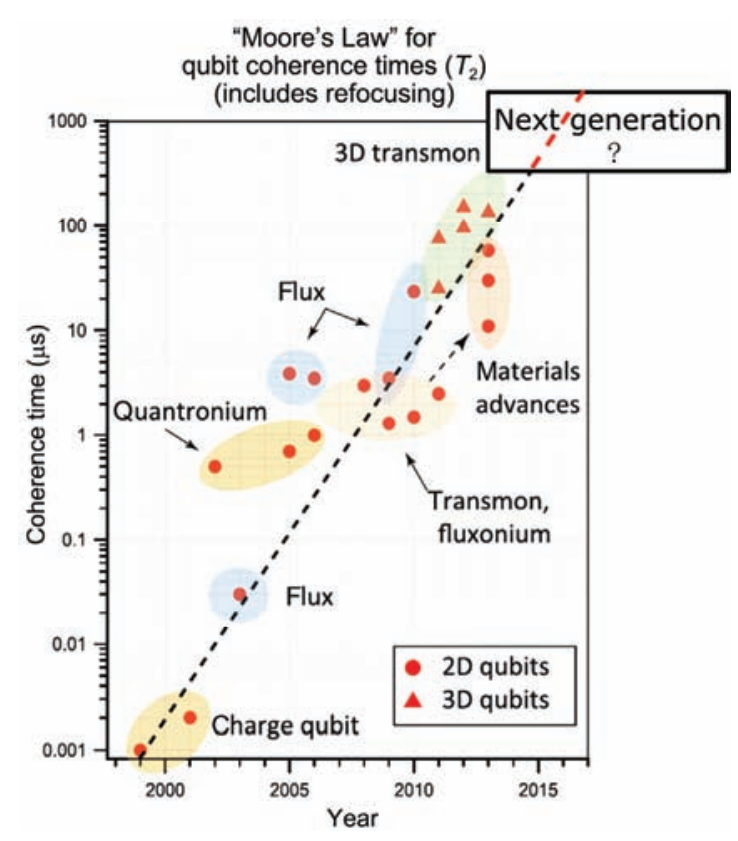

图 11 (网络版彩色)超导量子比特退相干时间发展的“摩尔定律”(根 据文献[42]修改)

Figure 11 (Color online) The "Moore's low" for the coherence time of superconducting qubits (modified from Ref. [42])

\section{参考文献}

1 Feynman R P. Simulating physics with computers. Int J Theor Phys, 1982, 21: 467-488

2 Shor P. Polynomial-time algorithms for prime factorization and discrete logarithms on a quantum computer. SIAM J Comput, 1997, 26: 1484-1509 
Grover L K. Quantum mechanics helps in searching for a needle in a haystack. Phys Rev Lett, 1997, 79: 325-328

Shor P W. Scheme for reducing decoherence in quantum computer memory. Phys Rev A, 1995, 52: R2493-R2496

Steane A. Multiple-particle interference and quantum error correction. Proc R Soc London A: Math Phys Eng Sci, 1996, 452: 2551-2577

Knill E, Laflamme R. Theory of quantum error-correcting codes. Phys Rev A, 1997, 55: 900-911

Gottesman D. Stabilizer codes and quantum error correction. 1997, arXiv: quant-ph/9705052

Devoret M H, Schoelkopf R J. Superconducting circuits for quantum information: An outlook. Science, 2013, 339: 1169-1174

Mermin N D. Quantum Computer Science. Cambridge: Cambridge University Press, 2007

Nielsen M A, Chuang I L, Zhao Q C. (translated). Quantum Computation and Quantum Information (in Chinese). Beijing: Tsinghua University Press, 2003 [Nielsen M A, Chuang I L. 赵千川(译). 量子计算与量子信息. 北京：清华大学出版社, 2003]

DiVincenzo D P. The physical implementation of quantum computation. Fortschr Phys, 2000, 48: 771-783

Clarke J, Wilhelm F K. Superconducting quantum bits. Nature, 2008, 453: 1031-1042

Sank D T. Fast, accurate state measurement in superconducting qubits. Dissertation for Doctoral Degree. Santa Barbara: University of California, 2014

Nakamura Y, Pashkin Y A, Tsai J S. Coherent control of macroscopic quantum states in a single-cooper-pair box. Nature, 1999, 398: 786-788

Barends R, Kelly J, Megrant A, et al. Superconducting quantum circuits at the surface code threshold for fault tolerance. Nature, 2014, 508: 500-503

Likharev K K. Dynamics of Josephson Junctions and Circuits. New York: Gordon and Breach Science Publishers, 1986

Josephson B D. Possible new effects in superconductive tunneling. Phys Lett, 1962, 1: 251-253

Anderson P W, Rowell J M, Probable observation of the Josephson superconducting tunneling effect. Phys Rev Lett, 1963, 10: 230-232

Devoret M H, Wallraff A, Martinis J M. Superconducting qubits: A short review. 2004, arXiv: cond-mat/0411174

Makhlin Y, Schön G, Shnirman A. Quantum-state engineering with Josephson-junction devices. Rev Mod Phys, 2001, 73: 357-400

You J Q. Quantum computation with superconducting circuit (in Chinese). Physics, 2010, 39: 810-815 [游建强. 基于超导量子器件的 量子计算. 物理, 2010, 39: 810-815]

Mao G F, Yu Y. Superconducting quantum bits based on Josephson junctions (in Chinese). Progress Phys, 2007, 27: 34-42 [毛广丰，于 扬. 基于约瑟夫森器件的超导量子比特. 物理学进展, 2007, 27: 34-42]

Creedon D L, Reshitnyk Y, Farr W, et al. High $Q$-factor sapphire whispering gallery mode microwave resonator at single photon energies and millikelvin temperatures. Appl Phys Lett, 2011, 98: 222903

Vion D, Aassime A, Cottet A, et al. Manipulating the quantum state of an electrical circuit. Science, 2002, 296: 886-889

Koch J, Yu T M, Gambetta J, et al. Charge-insensitive qubit design derived from the Cooper pair box. Phys Rev A, 2007, 76: 042319

Chen Y, Neill C, Roushan P, et al. Qubit architecture with high coherence and fast tunable coupling. Phys Rev Lett, 2014, 113: 220502

Paik H, Schuster D I, Bishop L S, et al. Observation of high coherence in Josephson junction qubits measured in a three-dimensional circuit QED architecture. Phys Rev Lett, 2011, 107: 240501

Jeffrey E, Sank D, Mutus J Y, et al. Fast accurate state measurement with superconducting qubits. Phys Rev Lett, 2014, $112: 190504$

Kelly J, Barends R, Fowler A G, et al. State preservation by repetitive error detection in a superconducting quantum circuit. Nature, 2015, 519: 66-69

Song C, Xu K, Liu W, et al. 10-qubit entanglement and parallel logic operations with a superconducting circuit. 2017, arXiv: 1703.10302

Shankar S, Hatridge M, Leghtas Z, et al. Autonomously stabilized entanglement between two superconducting quantum bits. Nature, 2013, 504: 419-422

Reagor M, Pfaff W, Axline C, et al. Quantum memory with millisecond coherence in circuit QED. Phys Rev B, 2016, 94: 014506

Brecht T, Pfaff W, Wang C, et al. Multilayer microwave integrated quantum circuits for scalable quantum computing. NPJ Quantum Inf, 2016, 2: 16002

Manucharyan V E, Koch J, Glazman L I, et al. Fluxonium: Single cooper-pair circuit free of charge offsets. Science, 2009, 326: 113-116 Langenberg D N. Physical interpretation of the $\cos \phi$ term and implications for detectors. Rev Phys Appl, 1974, 9: 35-40

Pop I M, Geerlings K, Catelani G, et al. Coherent suppression of electromagnetic dissipation due to superconducting quasiparticles. Nature, 2014, 508: 369-372

Yan F, Gustavsson S, Kamal A, et al. The flux qubit revisited to enhance coherence and reproducibility. Nat Commu, 2016, 7: 12964

You J Q, Hu X, Ashhab S, et al. Low-decoherence flux qubit. Phys Rev B, 2007, 75: 140515

Steffen M, Ansmann M, McDermott R, et al. State tomography of capacitively shunted phase qubits with high fidelity. Phys Rev Lett, 2006, 97: 050502

Steffen M, Kumar S, DiVincenzo D P, et al. High-coherence hybrid superconducting qubit. Phys Rev Lett, 2010, 105: 100502

Birenbaum J. The C-shunt flux qubit: A new generation of superconducting flux qubit. Dissertation for Doctoral Degree. Berkeley: University of California, 2014

Oliver W D, Welander P B. Materials in superconducting quantum bits. MRS Bull, 2013, 38: 816-825 


\title{
The road to long coherence time superconducting quantum bits
}

\author{
JIN YiRong* \& ZHENG DongNing \\ National Laboratory for Superconductivity, Beijing National Laboratory for Condensed Matter Physics, Institute of Physics, Chinese Academy of \\ Sciences, Beijing 100190, China \\ * Corresponding author, E-mail: jyr-king@iphy.ac.cn
}

Superconducting quantum computing is a solid state quantum computing technology based on Josephson junction circuits. It becomes one of the most promising candidates for realizing practical quantum computer/simulator, because of its scalability, easy to coupling and control, compatibility to semiconductor technologies, etc. As the complicated electromagnetic environment in solid state metal and insulator, superconducting quantum bits (qubits) suffered from shot coherence time since its early development. In this review, we described some recently developed superconducting qubit structures, including Transmon/Xmon, Fluxonium and C-shunt flux qubit. Although these new qubit structures have very different circuit parameters and energy scales, they all find their way to effectively suppress environmental noises. As a result, their coherence time were improved significantly.

Transmon, a transmission-line shunted plasma oscillation qubit, was now among the most popular structures for building a multi-qubit superconducting quantum circuit. It introduced a relatively large shunt capacitor to remarkably reduce the charge dispersion. Although the non-linearity is also decreased, by operating in a properly chosen $E_{J} / E_{c}$, transmon can greatly reduce the charge noise while only sacrificing a small amount of non-linearity.

Xmon can be considered as a modified version of transmon. It is designed and optimized for multi-qubit operation and measurement with separate $X / Y$ and $Z$ control lines for each qubit, and a readout bus (a transmission line or a low- $Q$ bandpass filter) for simultaneous readout of all the qubits.

Fluxonium provide another solution for suppressing the charge noise in Cooper-pair box (CPB). It introduces a large shunt inductance to filter low-frequency offset charge in the small capacitor of the phase-slip junction (the small junction). To keep the qubit operating in the charge region, the shunt inductor should be large enough to keep the impedance larger than the superconducting impedance quantum $R_{Q^{\sim}} 1 \mathrm{k} \Omega$. As a result, the inductance can only be provided by a large series of Josephson junctions. Fluxonium keeps the advantage of high anharmonicity of CPB. In addition, it introduces another degree of freedom: external flux $\Phi_{\text {ext. }}$ Quasiparticles (electrons or holes) tunneling at the small junction add coherently to produce a " $1+\cos \varphi$ " term. As a result, when $\Phi_{\text {ext }}=\Phi_{0} / 2$, dissipation caused by quasiparticle tunneling is suppressed and the energy relaxation time $\left(T_{1}\right)$ increases drastically.

C-shunt flux qubit (CSFQ) decreases $\alpha$ and $E_{J}$ to suppress flux noise in the qubit. While the charge noise becomes the dominant noise source, a large shunt capacitor is added to suppress the charge noise, similar to that in a transmon qubit. It is worth to notice that in CSFQ, the $\alpha$ decreases to below but near $1 / 2$. As a result, the double-well potential no longer exists. The energy structure become similar to transmon, with the anharmonicity be opposite. CSFQ can get a much improved coherence time, while its anharmonicity can be larger than that of transmon.

Those new superconducting qubit structures provide different solutions to improve the coherence time. Although it looks much different in circuit models and parameters, they have ideas in common, and may act as guides to other novel designs with longer coherence time. We can summarize as below. (1) The qubit circuit should be free of small superconducting islands. Charge fluctuation in small islands is a major source of decoherence. As a result, increase the area of the islands, that is, increase the capacitance becomes a straight forward way to suppress the charge noise. In Fluxonium, the small junction is connected with a large series of large junctions that there is also no small islands in the whole loop. (2) Remove superconducting loops in the qubit circuit, or minimize the loop size and loop current to suppress flux noise. (3) Bias the phase difference of the small junction to $\pi$, where dissipation caused by quasiparticle tunneling can be suppressed coherently. (4) Decrease the coupling bandwidth of qubit to the environment. In a circuit-QED readout scheme, a high- $Q$ readout resonator that dispersively coupled to the qubit protects the qubit from exposed to the electromagnetic environment of the readout circuit. A "Purcell filter" can also be added to suppress such decoherence further.

In conclusion, we overviewed some qubit engineering techniques or ideas to improve the coherence time, which is a very important figure for building practical quantum computer/simulator. We hope that someone can combine and optimize these ideas, while interact with developments in materials and micro-fabrication technologies, to develop novel new generation superconducting qubit structures in the near future.

quantum computing, superconducting qubit, coherence time, Josephson junction

doi: 10.1360/N972017-00715 